\title{
CONTRIBUTO PARA O ESTUDO DE COMPETÊNCIA CULTURAL EM SAÚDE MENTAL DE ENFERMEIROS EM PORTUGAL'1
}

\author{
| Alexandre Bastos Fernandes²; Ana Paula Monteiro ${ }^{3}$ |
}

\section{RESUMO}

CONTEXTO: A competência e sensibilidade cultural dos Enfermeiros são dimensões estruturais para a eficácia e qualidade dos cuidados de saúde mental.

OBJECTIVOS: Esta investigação teve como objectivo a avaliação da competência multicultural em saúde mental numa amostra de 306 enfermeiros, utilizando a versão portuguesa da Multicultural Mental Health Awareness Scale (Khawaja, Gomes \&Turner, 2008).

METODOLOGIA: Após um processo de tradução e validação transcultural, a versão portuguesa, conjuntamente com um questionário sociodemográfico, foi aplicada a uma amostra de 306 Enfermeiros, com uma média de idades de 35,4 anos e um desvio padrão de 9,8 anos; $25,2 \%$ do sexo feminino e $74,8 \%$ do sexo masculino, a trabalhar em serviços diversificados. A colheita de dados foi realizada online tendo sido respeitados os critérios éticos previstos para este procedimento.

RESULTADOS: Dos inquiridos, $75.8 \%$ reportaram ter contacto directo com situações de multiculturalidade nos seus contextos profissionais. Globalmente, os scores obtidos com a aplicação da MMHAS demonstram que os enfermeiros participantes no estudo evidenciam níveis pouco elevados de Competência Cultural em Saúde Mental. No entanto, os enfermeiros que tiveram formação prévia na área da multiculturalidade em saúde, revelam níveis significativamente mais elevados de Competência cultural, no score global e em todas as dimensões da escala. Os enfermeiros especialistas apresentam níveis mais elevados de Competência cultural em saúde mental.

CONCLUSÕES: Este estudo evidencia a necessidade de programas estruturados de formação multicultural em saúde mental dirigidos a enfermeiros em Portugal, sobretudo na formação inicial e formação contínua em enfermagem.

\section{PALAVRAS-CHAVE: Saúde mental; Enfermagem transcultural; Competência cultural}

\section{RESUMEN}

“Contribución al estudio de la competencia cultural en enfermeros de salud mental en Portugal"

CONTEXTO: La competencia cultural de los enfermeros es fundamental para la eficacia y la calidad de la atención de salud mental. OBJECTIVOS: Evaluar la competencia multicultural en salud mental en una muestra de 306 enfermeros, utilizando la Escala de Conciencia Multicultural de Salud Mental - MMHAS (Khawaja, Gomes \& Turner, 2008).

METODOS: Después de un proceso de traducción y validación culturales, la versión en Portugués, junto con un cuestionario sociodemográfico, se aplicó a una muestra de 306 enfermeros, con una edad media de 35,4 años y desviación estándar de 9,8 años; $25,2 \%$ mujeres y $74,8 \%$ hombres, que trabajan en servicios de salud diversificados. La coleta de datos se llevó a cabo online, respetando los requisitos éticos para este procedimiento.

RESULTADOS: 75,8\% de los inquiridos habia tenido contacto directo con situaciones de multiculturalidad en sus contextos profesionales. Los resultados obtenidos con la aplicación de MMHAS muestran que los participantes tienen una baja competencia cultural en salud mental. Sin embargo, los enfermeros que han tenido una formación previa en multiculturalidad muestran niveles significativamente más altos de competencia cultural en salud mental, globalmente y en todas las dimensiones de la escala (conciencia cultural, conocimiento y las habilidades culturales). Enfermeros especializadas tienen niveles más elevados de competencia cultural en salud mental.

CONCLUSIONES: Este estudio evidencia la necesidad de programas estructurados de formación multicultural en Portugal, en la formación inicial y en la formación avanzada de Enfermeria.

\section{DESCRIPTORES: Salud mental; Enfermería transcultural; Competencia cultural}

\section{ABSTRACT \\ “Contribution to the study of nurses' competences on mental health in Portugal"}

BACKGROUND: The benefits of integrating cultural competence into health care have been well established, especially in the area of mental health nursing.

AIMS: This research aimed to evaluate the multicultural competence in mental health in a sample of 306 nurses, using the Portuguese version of Multicultural Mental Health Awareness Scale - MMHAS (Khawaja, Gomes \& Turner 2008).

METHODS: After a process of translation and cultural validation, the Portuguese version, together with a socio-demographic questionnaire, was applied to a sample of 306 nurses, with a mean age of 35.4 years and standard deviation of 9.8 years; $25.2 \%$ female and $74.8 \%$ male, working in diversified services. The data collection was conducted online, and ethical criteria for this procedure have been met.

RESULTS: $75.8 \%$ of the participants reported direct contact with situations of multiculturalism in their professional contexts. Overall, the scores obtained with the application of MMHAS show that participants in the study have low levels of multicultural competences in mental health. However, nurses who have had previous multicultural training have significantly higher levels of cultural competence in mental health, in all dimensions of the scale (Cultural awareness, cultural knowledge and cultural skills/abilities).

CONCLUSIONS: This study highlights the need for structured programs in multicultural training in mental health nurses in Portugal initial training and advanced training in culturally sensitive healthcare practice.

\section{KEYWORDS: Mental health; Transcultural nursing; Cultural competency}

Submetido em 28-02-2015

Aceite em 30-09-2015

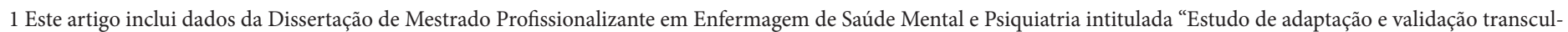

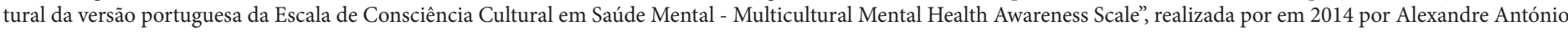
Bastos Fernandes sob orientação da Profa Doutora Ana Paula Teixeira de Almeida Vieira Monteiro.

2 Mestre em Enfermagem de Saúde Mental e Psiquiatria; Enfermeiro no Centro Hospitalar e Universitário de Coimbra, Portugal, bastos.fernandes@gmail.com

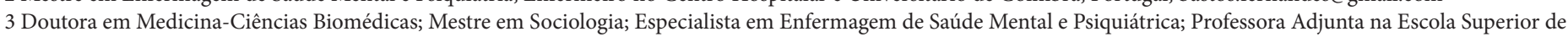
Enfermagem de Coimbra, Rua 5 de Outubro - Apartado 55, 3001-901 Coimbra, Portugal, anapaula@esenfc.pt

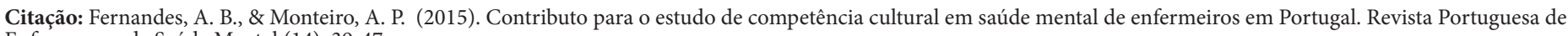
Enfermagem de Saúde Mental (14), 39-47. 


\section{INTRODUÇÃO}

A competência e sensibilidade cultural dos Enfermeiros são dimensões estruturais para a eficácia e qualidade dos cuidados de Enfermagem, sobretudo na área de enfermagem de saúde mental (Monteiro, 2009; Ordem dos Enfermeiros, 2010). Em Portugal não existem instrumentos validados para enfermeiros portugueses que permitam avaliar a competência cultural em saúde mental. Alguns estudos disponíveis focando as condições de saúde e a vulnerabilidade de alguns grupos de imigrantes específicos, bem como as dificuldades de acesso aos cuidados de saúde por parte de populações imigrantes em Portugal, apontam para a necessidade de formação dos técnicos de saúde ao nível das competências culturais (Moleiro e Gonçalves, 2010; Monteiro, 2011).

Na literatura, a noção de competência cultural em saúde tem sido objecto de uma multiplicidade de definições e modelos teóricos, mas pode ser sintetizada como a capacidade trabalhar com eficácia em situações culturalmente diferenciadas (Bean, 2006). Desde a década de setenta do século XX que a investigação teórica e clínica em enfermagem se debruça sobre o conceito de competência cultural nas suas múltiplas dimensões (Leininger, 1990; Giger \& Davidhizar, 2002; Campinha-Bacote,2003). O pressuposto metodológico de concepção de competência cultural em enfermagem nos processos de cuidar expressa-se através de uma aproximação etnográfica à experiência vivida das pessoas alvo dos cuidados de enfermagem, quer seja nos seus processos de transição vivencial, quer nos contextos de saúdedoença. Nesta perspectiva, podemos considerar que a conceptualização do cuidado multicultural na prática clínica, integrando os contributos da sociologia e da antropologia da saúde através dos constructos teóricos da etnoenfermagem (Leininger, 1990), foi uma conquista pioneira das ciências de enfermagem, que depois foi reapropriada e reconfigurada por outras áreas científicas afins nos domínios da saúde. De facto, a afirmação da enfermagem transcultural resultou em grande parte da formação de Madeleine Leininger em enfermagem psiquiátrica e do seu trabalho clínico num serviço de pedopsiquiatria. Neste contexto clínico, compreendeu que existia uma profunda lacuna na compreensão dos factores culturais que influenciavam o comportamento das crianças que estavam sob o seu cuidado. E foi esta necessidade de compreensão aprofundada tendo em conta o background cultural, que permitiu integrar nos cuidados de enfermagem a experiência subjectiva e social da doença mental, expressa num diálogo incessante entre o corpo e a cultura, em que os "idiomas culturais de sofrimento" são específicos (Kleinmam,1988; Helman, 2003). Os próprios itinerários de busca de ajuda para o sofrimento emocional ou para a perturbação psiquiátrica estão imbrincados de metáforas culturais que é preciso compreender "a partir de dentro" (Kirmayer, 2004; Monteiro e Mendes, 2013). Abreu (2008) define competência cultural como um processo contínuo de consciencialização cultural, conhecimento, habilidade, interacção e sensibilidade entre os cuidadores e as realidades em que trabalham. Todos os modelos de competência cultural em profissionais de saúde mental e partilham a noção essencial de que a competência cultural é um processo pluridimensional, com uma forte componente relacional.

Na vertente dos modelos multiculturais em saúde mental, sobretudo em contextos de psicoterapia e aconselhamento, o modelo tripartido de competência multicultural (Sue, Arredondo \& McDavis, 1992) tende a organizar-se em torno de três eixos fundamentais na sua conceptualização:1-Consciência Cultural; 2-Conhecimento cultural e 3-Habilidades/competências práticas culturais. De entre estas três dimensões integradoras, o conceito de Consciência Cultural consiste num processo de autoconhecimento que engloba a identificação dos próprios preconceitos e pressupostos relativamente aos outros. Desta forma, a Consciência Cultural integra a autoconsciência das atitudes e crenças do profissional de saúde relativamente a indivíduos de minorias étnicas e outros grupos culturais, assim como um conhecimento integrador sobre a sua própria origem cultural. Os profissionais de saúde mental devem compreender de que forma as suas próprias origens culturais, experiências pessoais e percursos existenciais influenciam valores, atitudes e enviesamentos relativamente aos mecanismos de avaliação dos processos de saúde e doença face a pessoas de outras culturas. Isto implica que os profissionais tenham também um conhecimento específico sobre a sua própria etnia e herança cultural e sobre a forma como esse background cultural afecta pessoal e profissionalmente as suas definições e possíveis enviesamentos sobre normalidade/disfuncionalidade e o processo terapêutico. A segunda dimensão, o Conhecimento Cultural, consiste no processo de procura e aquisição de conhecimentos sobre a diversidade cultural e as especificidades de grupos étnicos minoritários, nomeadamente sobre crenças relacionadas com saúde e valores culturais, incidência de doença e prevalência e eficácia de intervenções terapêuticas (Campinha-Bacote, 2002; Sue \& Sue, 2003). 
O conhecimento cultural representa, portanto, a compreensão aprofundada que o profissional de saúde vai construindo sobre os grupos culturais minoritários com quem trabalha e a compreensão das influências sociopolíticas exercidas sobre esses grupos. De resto, quanto maior a profundidade do conhecimento dos profissionais de saúde sobre as especificidades culturais de diversos grupos minoritários, maior a sua eficácia na prestação de cuidados de saúde culturalmente congruentes. O profissional culturalmente competente deve ter conhecimento claro e explícito e compreensão das características genéricas de indivíduos de diversas origens étnicas, raciais e socioeconómicas. Esta abordagem implica também o conhecimento das barreiras institucionais que dificultam a acessibilidade aos serviços de saúde mental. Por último, a dimensão Competência/ habilidades culturais, ou seja, de competências práticas multiculturais, reflecte a capacidade de pôr em prática a consciência e conhecimento multicultural, nas interacções com indivíduos culturalmente diversos. Implica competências técnicas de comunicação intercultural, capacidade para abordagens específicas de avaliação, intervenção e estratégias utilizadas no trabalho com grupos minoritários. Consiste também na capacidade de integrar factores culturais nos diagnósticos clínicos e na implementação de cuidados de enfermagem. Um estudo realizado por Monteiro e Mendes (2013) evidenciou a dificuldade de estabelecer diagnósticos clínicos em contextos de diversidade cultural, particularmente em unidades de psiquiatria. Os enfermeiros em contextos de praxis clínica em unidades de psiquiatria tendem a incorporar o modelo biomédico dominante. No entanto, surgem dificuldades interpretativas, que são objecto de interrogação crítica, face aos comportamentos aparentemente disfuncionais dos doentes. As dimensões socioculturais, numa perspectiva sistémica, podem explicar algumas variações de padrões de saúde e morbilidade psiquiátrica em populações imigrantes ou grupos étnicos minoritários. Por outro lado, as especificidades culturais são determinantes na forma como estas variações se manifestam ou são percepcionadas e interpretadas pelos próprios sujeitos que as vivenciam e pelas sociedades de acolhimento (Monteiro, 2011).

A sensibilidade cultural nos cuidados é especialmente relevante em enfermagem de saúde mental e em enfermagem psiquiátrica, em que o processo de relação terapêutica implica uma construção baseada no respeito incondicional do outro e numa compreensão empática em profundidade (Lazure,1995).
O desenvolvimento de competências culturais, da sensibilidade face à diversidade cultural e da habilidade de promover uma dinâmica assistencial apropriada a essa diferença, abrange todo o processo de assistência em enfermagem - no diagnóstico, no planeamento da intervenção, na implementação e na avaliação (Abreu, 2008). A formação específica nesta área é escassa em Portugal e pouca atenção tem sido dispensada na formação e desenvolvimento de competências multiculturais sistemáticas entre profissionais de saúde portugueses na área da saúde mental (Monteiro, 2009; Moleiro e Gonçalves, 2010). Não existem quaisquer estudos estruturados sobre os níveis de competência multicultural em enfermeiros portugueses nem sobre os factores que condicionam essas competências na área da saúde mental.

\section{METODOLOGIA}

A investigação, de natureza quantitativa, do tipo descritivo-correlacional, teve como principal objectivo avaliar os níveis de competência multicultural numa amostra de enfermeiros portugueses a trabalhar em diferentes contextos profissionais. Foi utilizada a versão Portuguesa da Multicultural Mental Health Awareness Scale - MMHAS (Khawaja, Gomez \& Turner, 2008). A versão original, de autopreenchimento, foi criada para avaliar o impacto de um programa de formação em competência cultural em saúde mental no Queensland Transcultural Mental Health Center (QTMHC) tendo como base o modelo tripartido de competência cultural (Sue, Bernier, Durran, Feinberg, Pedersen, Smith et al, 1982) e integra três dimensões: Consciência Cultural, Conhecimento Cultural e Competências/habilidades culturais práticas. Foi realizado um estudo de adaptação transcultural e validação psicométrica. A versão Portuguesa - Escala de Competência Multicultural em Saúde Mental (Fernandes, 2014) apresentou excelentes propriedades psicométricas com uma forte consistência interna (alfa de Cronbach de 0.958). Da análise factorial emergiram três factores bem definidos que integram dimensões de competência cultural coincidentes com os factores definidos na escala original - Consciência Cultural, Conhecimento Cultural e Competências/ habilidades culturais práticas, responsáveis por $59,51 \%$ da variância total. O questionário foi disponibilizado online através do LimeService / LimeSurvey de novembro de 2013 a fevereiro de 2014. Os participantes foram convidados a participar por email, redes sociais e contactos profissionais. 
As respostas referentes ao serviço onde trabalhavam foram bastante dispersas, sendo mais frequentes as consultas externas (35.7\%), os serviços de internamento (23.5\%), centros de saúde (13.7\%), os serviços de urgência (9.4\%) e instituições para idosos (6. 8\%). Uma parte significativa dos inquiridos (80.4\%) referiu não possuir formação específica na área da multiculturalidade. No entanto, a maioria (75.8\%) afirmou que na sua actividade profissional tinha contacto com utentes de grupos culturais minoritários (Quadro 2).

Quadro 2 - Características Profissionais da Amostra

\begin{tabular}{|c|c|c|}
\hline & $\mathrm{n}$ & $\%$ \\
\hline Categoria profissional & 300 & 98.0 \\
Enfermeiro(a) & 1 & 0.3 \\
Enfermeiro Graduado & 4 & 1.3 \\
Enfermeiro Especialista & 1 & 0.3 \\
Sem actividade & & \\
Experiência profissional (anos) & 82 & 26.8 \\
{$[5$} & 67 & 21.9 \\
{$[5-10[$} & 46 & 15.0 \\
{$[10-15[$} & 27 & 8.8 \\
{$[15-20[$} & 37 & 12.1 \\
{$[20-25[$} & 14 & 8.5 \\
{$[25-30[$} & 4 & 4.6 \\
{$[30-35[$} & & 1.3 \\
$\geq 35$ & & \\
x $12.23 ;$ Md $=10.00 ; s=9.79 ;$ & 60 & 19.6 \\
xmin $=0.00 ;$ xmáx $42.00 ; \mathrm{p}=0.000$ & 246 & 80.4 \\
Formação na área da multiculturalidade & & \\
Sim & & \\
Não & 232 & 75.8 \\
Contacto com utentes de grupos & 74 & 24.2 \\
culturais minoritários & & \\
Sim & & \\
Não & & \\
\hline
\end{tabular}

Os resultados do Quadro 3 foram obtidos com base na aplicação da versão portuguesa da Escala de Competências Multiculturais em Saúde Mental (MMHAS). Como se constata pelos resultados, principalmente os valores da posição relativa da média, os elementos da amostra evidenciaram níveis mais elevados de competência cultural em saúde mental na dimensão consciência cultural e níveis mais baixos na dimensão conhecimento cultural. Globalmente, o valor médio situou-se nos 35.00\% da amplitude do intervalo de avaliação. A dispersão relativa foi semelhante nas três dimensões e no global e a distribuição de frequências afastou-se significativamente da curva normal em todas as dimensões e, também, no global da escala. Considerando baixa a posição relativa das médias, face à amplitude dos respectivos intervalos de medida, podemos afirmar que os enfermeiros inquiridos evidenciaram possuir níveis pouco elevados de competência cultural em saúde mental.
Quadro 3 - Resultados da Avaliação da Escala MMHAS (Versão Portuguesa)

\begin{tabular}{|l|l|l|l|l|l|l|l|}
\hline $\begin{array}{l}\text { Escala } \\
\text { MMHAS }\end{array}$ & $\mathrm{x}$ & $\mathrm{Md}$ & $\mathrm{S}$ & $\mathrm{xmin}$ & xmáx & $\begin{array}{l}\text { Posição } \\
\text { relativa da } \\
\text { média (\%) }\end{array}$ & $\mathrm{p}$ \\
\hline Consciência & 37.70 & 37.00 & 8.91 & 19.00 & 64.00 & 47.50 & 0.010 \\
\hline Conhecimento & 20.11 & 20.00 & 5.52 & 12.00 & 51.00 & 20.70 & 0.000 \\
\hline Competências & 26.18 & 25.00 & 6.12 & 14.00 & 44.00 & 34.50 & 0.000 \\
\hline Global & 83.99 & 82.00 & 17.61 & 49.00 & 158.00 & 35.00 & 0.005 \\
\hline
\end{tabular}

Para analisar a relação entre factores sociodemográficos/profissionais e os níveis de competência cultural avaliada através da MMHAS (versão portuguesa) foi realizado um estudo descritivo-correlacional. Relativamente às diferenças de género, a comparação dos resultados obtidos pelos inquiridos do género feminino e pelos do género masculino, aplicando o teste $U$ de Mann-Whitney, permitiu obter os resultados que constituem o Quadro 4. Observa-se a existência de diferenças estatisticamente significativas ao nível do conhecimento cultural ( $\mathrm{p}=0.014)$. Comparando os valores das medidas de tendência central (média ordinal, média e mediana) podemos afirmar que os enfermeiros do género feminino tenderam a revelar um nível mais elevado de conhecimento cultural em saúde mental que os do género masculino.

Quadro 4 - Competência Cultural em Saúde Mental em Função do Género

\begin{tabular}{|c|c|c|c|c|c|c|}
\hline $\begin{array}{l}\text { MMHAS } \\
\text { Género }\end{array}$ & $\mathrm{n}$ & Xord & $X$ & Md & z & $\mathrm{p}$ \\
\hline $\begin{array}{l}\text { Consciência } \\
\\
\text { Feminino } \\
\text { Masculino } \\
\text { Conhecimento } \\
\text { Feminino } \\
\text { Masculino } \\
\text { Competências } \\
\text { Feminino } \\
\text { Masculino } \\
\text { Global } \quad \text { Feminino } \\
\\
\text { Masculino }\end{array}$ & $\begin{array}{l}77 \\
229 \\
77 \\
229 \\
77 \\
229 \\
77 \\
229\end{array}$ & $\begin{array}{l}151.62 \\
154.13 \\
174.84 \\
146.32 \\
162.65 \\
150.42 \\
160.92 \\
151.01\end{array}$ & $\begin{array}{l}37.35 \\
37.82 \\
21.64 \\
19.60 \\
\\
27.08 \\
25.88 \\
\\
86.06 \\
83.30\end{array}$ & $\begin{array}{l}39.00 \\
37.00 \\
21.00 \\
19.00 \\
25.00 \\
25.00 \\
\\
85.00 \\
82.00\end{array}$ & $\begin{array}{l}-1.051 \\
-0.850\end{array}$ & 0.395 \\
\hline
\end{tabular}

Para o avaliar a relação entre a competência cultural em saúde mental e a escolaridade, optámos pelo teste Kruskal-Wallis. Como podemos constatar pelos dados que apresentamos no Quadro 5 existem diferenças estatisticamente significativas em todas as dimensões e no global da escala $(\mathrm{p}<0.050)$. Na escala global de competência cultural em saúde mental, observaram-se, igualmente, diferenças estatisticamente significativas relativamente à escolaridade dos participantes $(\mathrm{p}=0.000)$. Os enfermeiros que possuíam Bacharelato/Licenciatura apresentam em média scores mais baixos (81.01). 
Os enfermeiros com a Especialidade em Enfermagem de Saúde Mental e Psiquiátrica (ESMP) evidenciam, em média, com significância estatística, scores mais elevados de competência cultural, quer no global da escala (92.60), quer nas dimensões consciência cultural - 42,06 ( $\mathrm{p}=0.001)$; conhecimento cultural em saúde mental - 21,85 ( $\mathrm{p}=0.021)$ e competências práticas em saúde mental - 28.68 ( $\mathrm{p}=0.003)$.

Os inquiridos com Bacharelato/Licenciatura foram os que apresentaram, com significância estatística, em média, scores menos elevados - no global da escala e em todas as dimensões.

Ao nível da dimensão competência/Habilidades práticas observaram-se diferenças estatisticamente significativas entre os vários níveis de formação académica dos participantes $(p=0.003)$. Os enfermeiros Especialistas (em Enfermagem de Saúde Mental e Psiquiátrica ou especialistas outra área), evidenciaram maiores competências práticas, com scores médios de respectivamente, 28,68 e 28,52. Os enfermeiros com Bacharelato/Licenciatura ou Mestrado/Doutoramento apresentam em média, scores mais baixos (respectivamente de 25.40 e 25.26).

Quadro 5 - Competência Cultural em Saúde Mental em Função da Formação Académica

\begin{tabular}{|l|l|l|l|l|l|l|}
\hline $\begin{array}{l}\text { Score MMHAS /Escolari- } \\
\text { dade }\end{array}$ & $\mathrm{n}$ & Xord & $\mathrm{X}$ & $\mathrm{Md}$ & $\mathrm{X}^{2}$ & $\mathrm{p}$ \\
\hline Consciência & & & & & 17.656 & 0.001 \\
Bacharelato/Licenciatura & 192 & 139.85 & 36.17 & 36.00 & & \\
Especialidade ESMP & 47 & 195.80 & 42.06 & 41.00 & & \\
Outra Especialidade & 29 & 178.38 & 40.79 & 40.00 & & \\
Mestrado/Doutoramento & 38 & 151.17 & 37.71 & 37.50 & & \\
\hline Conhecimento & & & & & 9.687 & 0.021 \\
Bacharelato/Licenciatura & 192 & 143.24 & 19.44 & 19.00 & & \\
Especialidade ESMP & 47 & 185.39 & 21.85 & 22.00 & & \\
Outra Especialidade & 29 & 169.59 & 20.48 & 21.00 & & \\
Mestrado/Doutoramento & 38 & 153.61 & 21.05 & 20.00 & & \\
\hline Competências/habilidades & & & & & 14.058 & 0.003 \\
práticas & & & & & & \\
Bacharelato/Licenciatura & 192 & 143.71 & 25.40 & 24.00 & & \\
Especialidade ESMP & 47 & 185.12 & 28.68 & 28.00 & & \\
Outra Especialidade & 29 & 188.00 & 28.52 & 28.00 & & \\
Mestrado/Doutoramento & 38 & 137.55 & 25.26 & 24.50 & & \\
\hline Global & & & & & 20.172 & 0.000 \\
Bacharelato/Licenciatura & 192 & 139.30 & 81.01 & 79.50 & & \\
Especialidade ESMP & 47 & 197.34 & 92.60 & 94.00 & & \\
Outra Especialidade & 29 & 184.28 & 89.79 & 87.00 & & \\
Mestrado/Doutoramento & 38 & 147.51 & 84.03 & 82.00 & & \\
\hline
\end{tabular}

A avaliação da relação entre a formação específica na área da multiculturalidade e a competência cultural em saúde mental foi realizada através da aplicação do teste U de Mann-Whitney (Quadro 6). Os resultados que constituem o quadro 6 permitem-nos verificar que todas as diferenças observadas são estatisticamente significativas $(\mathrm{p}<0.050)$.
A análise dos valores observados para as medidas de tendência central revela que os enfermeiros que tiveram formação na área da multiculturalidade revelaram melhor competência cultural em saúde mental no global da escala e em todas as dimensões avaliadas pela escala.

Quadro 6 - Competência Cultural em Função de Formação na Área da Multiculturalidade

\begin{tabular}{|c|l|l|l|l|l|l|}
\hline $\begin{array}{l}\text { MMHAS } \\
\text { Formação na área } \\
\text { da multiculturalidade }\end{array}$ & $\mathrm{n}$ & Xord & $\mathrm{X}$ & $\mathrm{Md}$ & $\mathrm{z}$ & $\mathrm{p}$ \\
\hline $\begin{array}{c}\text { Consciência Cultural } \\
\text { Sim }\end{array}$ & 60 & 214.55 & 43.97 & 44.00 & & \\
Não & 246 & 138.61 & 36.17 & 35.50 & & \\
$\begin{array}{c}\text { Conhecimento Cultural } \\
\text { Sim }\end{array}$ & 60 & 212.93 & 24.28 & 23.50 & & \\
Não & 246 & 139.01 & 19.09 & 19.00 & & \\
Competências práticas & 60 & 189.55 & 29.13 & 27.50 & & \\
Sim & 246 & 144.71 & 25.46 & 25.00 & & \\
Não & 60 & 214.33 & 97.38 & 96.50 & & \\
Sim & 246 & 138.66 & 80.73 & 79.50 & & \\
Global Não & & & & & & \\
\hline
\end{tabular}

A aplicação do teste U de Mann-Whitney permitiu, também, avaliar se a consciência cultural em saúde mental é diferente conforme o enfermeiro tem, ou não, contacto com utentes de grupos culturais minoritários (Quadro 7). Podemos verificar que existem diferenças estatisticamente significativas em duas das dimensões consciência cultural ( $p=0.009)$, conhecimento cultural $(\mathrm{p}=0.004)$ e no global da escala $(\mathrm{p}=0.003)$.

Quadro 7 - Consciência Cultural em Saúde Mental / Contacto com Utentes de Grupos Culturais Minoritários

\begin{tabular}{|c|l|l|l|l|l|l|}
\hline $\begin{array}{l}\text { Contacto com utentes } \\
\text { de grupos culturais } \\
\text { minoritários }\end{array}$ & $\mathrm{n}$ & Xord & $\mathrm{X}$ & $\mathrm{Md}$ & $\mathrm{z}$ & $\mathrm{p}$ \\
\hline $\begin{array}{c}\text { Consciência Cultural } \\
\text { Sim }\end{array}$ & 232 & 160.97 & 38.36 & 38.00 & -2.615 & 0.009 \\
Não & 74 & 130.09 & 35.65 & 34.00 & & \\
Conhecimento Cultural & 232 & 161.64 & 20.63 & 20.00 & & \\
Sim & 74 & 127.97 & 18.47 & 18.00 & & \\
Não & 232 & 158.95 & 26.61 & 25.00 & & \\
Competências práticas & 74 & 136.41 & 24.82 & 24.00 & & \\
Sim & & & & & -2.929 & 0.003 \\
Não & 232 & 161.86 & 85.60 & 85.00 & & \\
Sim & 74 & 127.28 & 78.95 & 75.00 & & \\
Não & & & & & & \\
\hline
\end{tabular}

\section{DISCUSSÃO DOS RESULTADOS}

De acordo com a literatura, os modelos de competência cultural em saúde mental tendem a organizar-se em torno de três eixos fundamentais na sua conceptualização - Consciência Cultural; Conhecimento cultural e Habilidades culturais/competências práticas e todos partilham a noção de que a competência cultural é um processo pluridimensional com uma forte componente relacional. 
O objectivo deste estudo foi avaliar os níveis de competência multicultural numa amostra de 306 enfermeiros portugueses a trabalhar em diferentes contextos profissionais, utilizando a versão Portuguesa da Multicultural Mental Health Awareness Scale - MMHAS (Khawaja, Gomez \& Turner, 2008) validada por Fernandes (2014) e um questionário sociodemográfico. Os questionários foram disponibilizados online e cumprindo requisitos éticos previstos para esta metodologia. A amostra foi constituída maioritariamente por enfermeiros jovens, predominantemente licenciados ou com bacharelato e maioritariamente do sexo masculino (74.8\%). Esta distribuição por género não corresponde aos dados sobre a constituição sociodemográfica dos enfermeiros em Portugal e pode ser explicada devido à colheita de dados ter sido realizada online.

Os resultados da aplicação da escala MMHAS versão portuguesa evidenciam que os inquiridos apresentam níveis pouco elevados de Competência cultural em saúde mental, uma vez que o valor médio se situou nos 35,00\%. O aparente deficit de competências multiculturais em saúde mental, consideradas essenciais para o cuidar integral em enfermagem, pode ter um profundo impacto na qualidade dos cuidados (Leininger, 1990).

A questão da saúde /doença está profundamente dependente de determinantes sociais e tem subjacente um conjunto de marcadores culturais, algo especialmente relevante em grupos étnicos minoritários. $\mathrm{Na}$ praxis clínica de enfermagem, nem sempre é bem compreendida esta questão-chave da multiculturalidade, quer na expressão do sofrimento emocional, quer na demarcação de sintomas, quer nas representações sobre o que é saúde/doença, o que tem implicações nas possibilidades de diagnóstico e intervenção e nos processos de relação terapêutica construída pelos enfermeiros (Kirmayer, 2004; Monteiro, 2011). No entanto, num mundo globalizado em que o fluxo de pessoas e etnias aumentou radicalmente, a diversidade cultural dos utilizadores dos serviços de saúde tende a aumentar e a tornar-se cada vez mais complexa (Monteiro e Mendes, 2013), o que se reflecte nos achados deste estudo $75.8 \%$ dos enfermeiros inquiridos reportaram ter contacto directo com situações de multiculturalidade nos seus contextos profissionais. Ou seja, apesar da necessidade concreta de prestar cuidados de enfermagem em contextos de multiculturalidade, paradoxalmente, uma parte significativa dos participantes revelou não ter competências adequadas.

A investigação realizada indicia que as competências multiculturais em saúde mental dos enfermeiros são mediadas por uma diversidade de factores.
Neste estudo, a escolaridade dos enfermeiros inquiridos influencia os níveis de competência cultural em saúde mental, uma vez que foram encontradas diferenças estatisticamente significativas em todas as dimensões da escala e no global. Os enfermeiros com Bacharelato/ Licenciatura foram os que obtiveram piores resultados, com níveis menos elevados de cotação na escala global e nas suas dimensões. Estes dados evidenciam a o impacto da diferenciação da formação avançada e a sua importância na competência multicultural em enfermagem.

$\mathrm{Na}$ dimensão Competência/habilidades práticas culturais, os resultados obtidos merecem uma apreciação, uma vez que os enfermeiros especialistas obtiveram melhores resultados do que os enfermeiros detentores do grau académico de Mestrado/Doutoramento. Estes dados devem ser lidos com cautela, mas podem indiciar que a formação e a praxis clínica especializada em contextos de multiculturalidade fornece aos enfermeiros competências específicas que os tornam mais competentes e mais capazes de prestar cuidados culturalmente sensíveis, independentemente da sua qualificação académica formal. De acordo com a literatura, a construção da Competência Cultural em Enfermagem inicia-se com a consciencialização sobre a forma como as próprias culturas de origem dos Enfermeiros afetam diferentes aspetos das suas vidas permitindo-lhes compreender a sua identidade cultural e o seu "olhar culturalmente centrado" na percepção da realidade, do mundo, do "outro", das relações de sociabilidade, de intersubjetividade e na prestação de cuidados. Pela análise dos resultados de cada dimensão pode concluirse que os inquiridos apresentam melhores resultados na dimensão Consciência Cultural do que na dimensão Competências práticas ou Conhecimento Cultural. Analisando a influência das variáveis sociodemográficas na Competência Cultural em saúde mental nos enfermeiros inquiridos, observou-se que os enfermeiros do género feminino apresentam uma melhor competência cultural na dimensão do Conhecimento cultural que os do sexo masculino.

A formação específica na área da multiculturalidade foi outra variável estudada e os resultados obtidos vão de encontro aos referentes teóricos disponíveis, uma vez que os enfermeiros que tiveram formação prévia na área da multiculturalidade revelam uma melhor competência cultural em saúde mental e apresentam diferenças estatisticamente significativas em todas as dimensões da escala e no global. 
A literatura reporta que a formação para a multiculturalidade dos profissionais de saúde melhora a acessibilidade e a eficácia dos cuidados de saúde prestados a grupos étnicos minoritários, aumentando a consciência, o conhecimento e as habilidades dos prestadores de cuidados e modificando as políticas e práticas das organizações de saúde (Truong, Paradies \& Priest, 2014). No nosso estudo, os enfermeiros que reportaram contacto com utentes de grupos culturais minoritários apresentam cotações mais elevadas nas dimensões consciência cultural e conhecimento cultural, bem como na escala global. $O$ facto de na dimensão competências/habilidades práticas não se encontrarem diferenças significativas, pode dever-se ao facto de o contacto com grupos culturais minoritários provocar nos enfermeiros uma maior percepção das dificuldades de realizar cuidados de enfermagem culturalmente sensíveis. Esta avaliação pode também dever-se às dificuldades práticas encontradas no relacionamento com grupos culturais minoritários no contexto dos cuidados. Como se trata do primeiro estudo realizado no nosso país, sugere-se que mais estudos sejam desenvolvidos para averiguar factores específicos que interferem com aas competências culturais em saúde mental de enfermeiros portugueses. As limitações do estudo, passam pelo facto de não existir mais nenhum instrumento de medida de competências multicultural de saúde mental validado para a população portuguesa que possa ser utilizado como comparação dos resultados obtidos. Por outro lado, este estudo baseia-se num questionário de autopercepção e auto-avaliação de competências, pelo que outros estudos podem utilizar metodologias diversas na óptica dos utilizadores dos cuidados permitindo validar a informação obtida.

Uma outra limitação é o facto de, na amostra deste estudo, a percentagem de elementos do género masculino, ser muito superior à do género feminino o que não é ilustrativo da população em estudo e poder implicar algum eventual viés nos resultados globais.

\section{CONCLUSÕES}

Os achados deste estudo indiciam que, globalmente, os enfermeiros inquiridos evidenciaram níveis pouco elevados de Competência cultural em saúde mental. Os participantes que tiveram formação prévia na área da multiculturalidade em saúde revelam níveis mais elevados de Competência cultural e apresentam diferenças estatisticamente significativas em todas as dimensões da escala e no global.
Os enfermeiros participantes com contacto com utentes de grupos culturais minoritários apresentam valores superiores de Competência cultural, nas dimensões Consciência Cultural e Conhecimento Cultural, bem como, no global; os enfermeiros do género feminino apresentam níveis mais elevados de Competência cultural na dimensão do Conhecimento cultural que os do sexo masculino; os enfermeiros com a especialidade em saúde mental e psiquiátrica apresentam níveis mais elevados de Competência cultural em saúde mental (nas três dimensões avaliadas).

A formação de enfermeiros e outros profissionais de saúde para prestar cuidados de saúde de qualidade numa sociedade cada vez mais multicultural e global exige uma abordagem abrangente, que enfatize a educação para a competência cultural na formação académica e na vida profissional. Como ficou evidenciado neste estudo, a formação na área da multiculturalidade é uma necessidade premente, demonstrada pelos níveis pouco elevados de competência cultural dos enfermeiros inquiridos, pelas lacunas encontradas e pelo contacto existente com grupos culturais minoritários.

Com os resultados alcançados, julgamos ter colaborado para uma melhor compreensão da importância da competência cultural em saúde mental, e para a prestação de cuidados de saúde culturalmente sensíveis e congruentes. Sugere-se, contudo, o incremento da investigação nesta temática, dado o número reduzido de estudos publicados em Portugal. É fundamental que os enfermeiros e outros técnicos de saúde sejam sensibilizados para as questões da multiculturalidade e para a específica formação na área. É igualmente importante que as instituições compreendam a necessidade de cuidados de saúde culturalmente competentes, fornecendo formação e criando condições para que possam ser desenvolvidos.

\section{REFERÊNCIAS BIBLIOGRÁFICAS}

Abreu, W. (2008). Transições e contextos transculturais: Contributos para a anamnese e recurso aos cuidados informais (2a Ed.). Coimbra: Formasau.

American Psychological Association (2013). Ethical principles of psychologists and code of conduct. Acedido a 8 de Junho de 2013. Disponível em http://www. apa.org/ethics/code/principles.pdf

Campinha-Bacote, J. (2003). The process of cultural competence in the delivery of healthcare services: A culturally competent model of care (4th edn.). Cincinnati: Transcultural C.A.R.E. Associates. 
Fernandes, A. (2014). Estudo de adaptação e validação transcultural da versão portuguesa da Escala de Consciência Cultural em Saúde Mental - Multicultural Mental Health Awareness Scale - MMHAS (Tese de Mestrado). Escola Superior de Enfermagem de Coimbra, Coimbra.

Giger, J., \& Davidhizar, R. (2002). The Giger and Davidhizar Transcultural Assessment Model. Journal of Transcultural Nursing,13(3), 185-188.

Giger, J. N., \& Davidhizar, R. (1995). Canadian transcultural nursing: Applying the Giger and Davidhizar model. Journal of Cultural Diversity, 4(1), 26-31.

Jeffreys, M. R. (2010). Teaching cultural competence in nursing and health care. New York: Springer Publishing Company.

Khawaja, N. G., Gomez, I. F., \& Turner, G. (2008). Development of the Multicultural Mental Health Awareness Scale. Acedido em http://eprints.qut.edu. au/16923/1/16923.pdf

Kirmayer, L. (2004). The cultural diversity of healing: Meaning, metaphor and mechanism. British Medical Bulletin, 69, 33-48.

Lazure, H. (1994). Viver a relação de ajuda: Abordagem teórica e prática de um critério de competência da enfermeira. Lisboa: Lusodidacta.

Leininger, M. (1990). Ethnomethods: The philosophic and epistemic bases to explicate transcultural nursing knowledge. Journal of Transcultural Nursing, 1(2), 40-51.
Leininger, M., \& McFarland, M. (2006). Culture care diversity and universality. A worldwide nursing theory. Boston: Jones and Barlet.

Moleiro, C., \& Gonçalves, M. (2010). Saúde na diversidade: Desenvolvimento de serviços de saúde mental sensíveis à cultura. Análise Psicológica, 3(18): 505-515. Monteiro, A. P. (2011). Migração e saúde mental (1 $1^{\text {a }}$ ed.() Viseu: PsicoSoma.

Monteiro, A. P., \& Mendes, A. C. (2013). Multicultural care in nursing - From the theoretical paradigm to the subjective experiences in clinical settings, Open Journal of Nursing, 3, 557-562.

Organização Mundial da Saúde (2001). Relatório mundial da saúde - Saúde mental: Nova concepção, nova esperança. Lisboa: Direcção Geral de Saúde.

Sue, D., Arredondo, P., \& McDavis, R. (1992). Multicultural counseling competencies and standards: A call to the profession. Journal of Counseling \& Development, 70, 477-486.

Sue, D. W., Bernier, J. E., Durran, A., Feinberg, L., Pedersen, P., Smith, E. J., \& Vasquez-Nuttall, E. (1982). Position paper: Cross-cultural counseling competencies. The Counseling Psychologist, 10, 45-52.

Sue, D. W., \& Sue D. (2003). Counseling the culturally diverse: Theory and practice (4th Ed.). New York: Wiley. Truong, M., Paradies, Y., \& Priest, M. (2014). Interventions to improve cultural competency in healthcare: A systematic review of reviews. BMC Health Services Research, 14, 99.

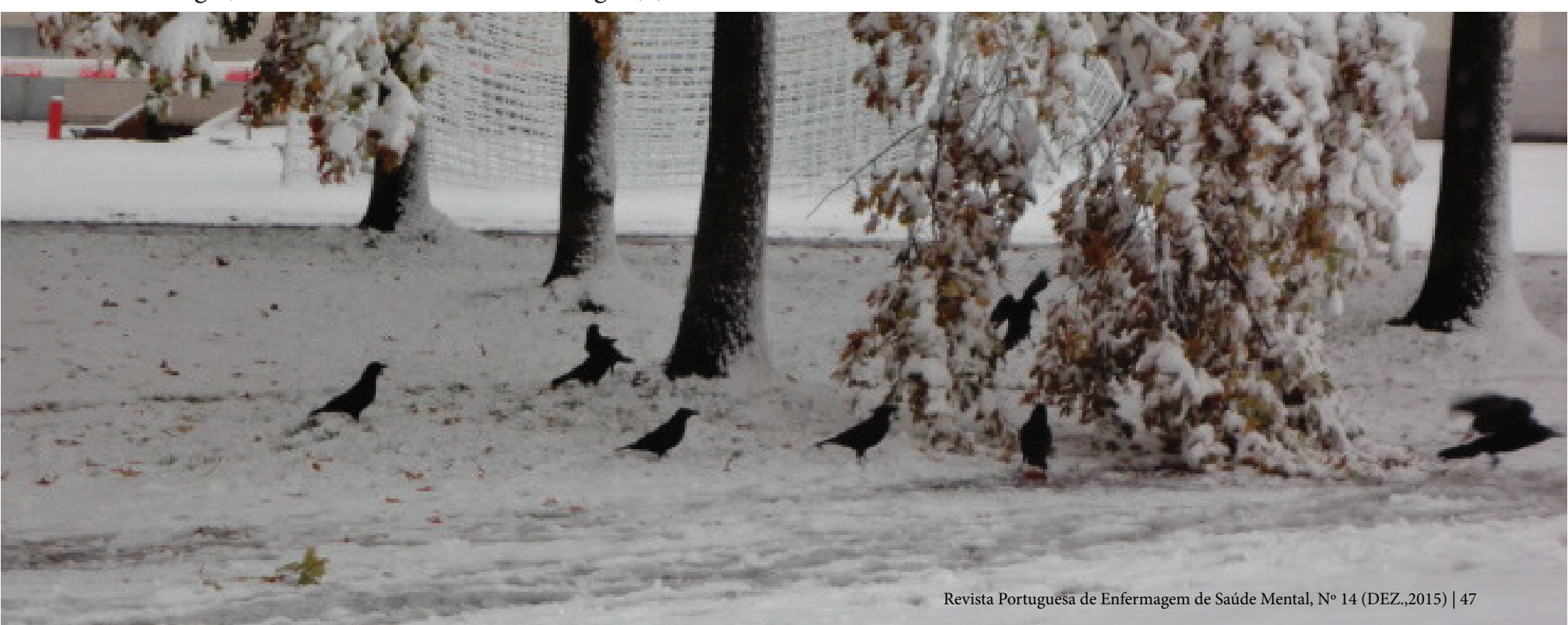

